\title{
DETERMINATION OF CRYSTAL STRUCTURE BY OPTICAL DIFFRACTION METHODS
}

IN some recent publications ${ }^{1-5}$, we have advocated the use of optical diffraction methods in crystalstructure determination, and have shown how they could have been applied to some known structures. The test of the methods, however, lies in their application to new problems, and this present communication is intended to give a review of what has been achieved so far.

Five structures have been successfully determined. For the simplest ones, optical methods, supplemented by knowledge of the stereochemistry of the molecule, proved to be entirely adequate; but for the others we have made use of such other information as would normally be available at the outset of an investigation. 'The characteristic of the methods is that no calculation is needed until the 'refinement' stage is reached.

The five structures are as follows:

(a) $p$-Di-isocyanobenzene ${ }^{6}$ (Fig. a). Space group $C 2 / m ; a=9 \cdot 80, b=7 \cdot 15, c=4.72 \mathrm{~A} ., \beta=97^{\circ} 11^{\prime}$. Molecules on $2 / m$.

(b) Diphenylene naphthacene (Fig. b). Space group $P 2_{1} / c ; a=11 \cdot 0, b=5 \cdot 15, c=19 \cdot 6 \mathrm{~A}$., $\beta=126^{\circ}$. Molecules on $\overline{1}$.

(c) Thiourea dioxide (Fig. c). Space group $P 2, n b$; $a=9.95, b=10.64, c=3.93 \mathrm{~A}$. Molecules in general positions.

(d) Di-p-anisyl nitric oxide (Fig. d). Space group Aba2 ; $a=7 \cdot 33, b=26 \cdot 8, c=6.08$ A. Molecules on 2 .

(e) Purpurogallin. Space group (effectively) $P 2_{1} 2_{1} 2$; $a=9 \cdot 78, b=24 \cdot 6, c=3 \cdot 65 \mathrm{~A}$. Molecules in general positions.

The problems are arranged roughly in order of complexity. The first one- $p$-di-isocyanobenzenecould have been readily solved by any method, but the solution by the Fourier-transform method was almost immediate. The factor of agreement with the atomic parameters derived ${ }^{6}$ was $0 \cdot 185$.

The second-diphenylene naphthacene-was of the same order of complexity as phthalocyanine ${ }^{1}$, and

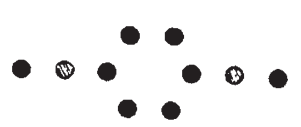

(a)

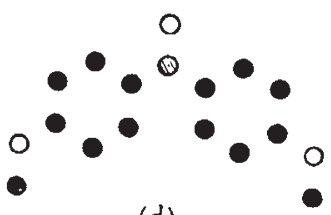

(d)

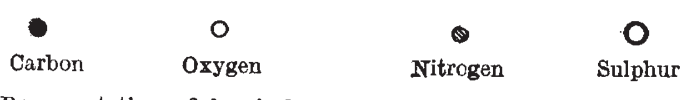

Representations of chemical molecules. (a) $p$-Di-isocyanobenzene. (b) Diphenylene naphthacene. (c) Thiourea dioxide. (d) Di-p-anisyl exactly similar methods were used. In addition, of the sixty-three signs determined by the methods described by Lipson and Taylor', only two were found to be incorrect. The factor of agreement given by the first parameters was $0 \cdot 28$.

The third structure-thiourea dioxide-was more difficult at the outset because, although the chemical molecule contains only six atoms (neglecting hydrogen), the chemical constitution was uncertain. Initially it was thought that the space group was Pmnb, and thus that the molecules lay on mirror planes; on this basis the signs of the structure factors were found by the Fourier-transform method ${ }^{3}$, the most likely form of molecule being assumed. Optical Fourier synthesis ${ }^{2,5}$, however, showed only the positions of the sulphur atoms, but by systematic exploration of sign combinations of some of the less certain terms, a recognizable representation of the complete molecule was obtained optically. It is now known that this structure is only approximate, the space group probably being $P 2_{1} n b$.

In the fourth structure-a free radical, di-p-anisyl nitric oxide-the stereochemistry was only approximately known. By trial of various possibilities, suggested by the Fourier-transform method and by packing considerations, a projection was found that gave reasonable agreement, and calculation showed that the factor of agreement was about $0 \cdot 35$.

The fifth structure-purpurogallin - is of a different standard of complexity from the others, and, since the methods used show clearly the value of the optical approach, it is dealt with in a separate communication?.

From these results, it would appear that the Fourier-transform method ${ }^{3}$ is the most powerful of the methods we have advocated, and is greatly helped by the modified 'fly's eye' device. Fourier synthesis ${ }^{2,5}$ is likely to be of much more limited application, unless it is possible greatly to extend the area (about $4 \frac{1}{2} \mathrm{in}$. in diameter) of the beam of parallel light available in the present apparatus.

We are grateful to Dr. I. G. Edmunds and Mr. A. Bennett, and to Mr. B. M. Hargreaves, of Messrs. Hardman and Holden, Ltd., for allowing us access to their data on diphenylene-naphthacene and thiourea dioxide respectively, and to Dr. A. Burawoy for providing us with a specimen of di-p-anisyl nitric oxide. We also wish to record our thanks to the Department of Scientific and Industrial Research and to the Council of the Royal Society for grants towards the building of the apparatus.

The methods and results will be described in greater detail elsewhere.

\section{A. W. Hanson \\ C. A. TAYLOR \\ H. LIPSON}

Physics Department, College of Technology,

Manchester 1. April 23.

${ }^{1}$ Taylor, C. A., and Lipson, H., Nature, 167, 869 (1951).

2 Hanson, A. W. Taylor, C. A., and Jipson, H., Nature, 168, 160 (1951).

${ }^{8}$ Lipson, H., and T'aylor, C. A., Acta Cryst., 4, 458 (1951).

"Hanson, A. W., and Lipson, H., Acta Cryst., 5, 145 (1952).

"Hanson, A. W., and Lipson, H., Acta Cryst. (in the press).

- Hulme, R., Acta Cryst., 5, 144 (1952).

${ }^{2}$ Taylor, C. A. (see following communication). 